\title{
Flexible Complementarity Solvers for Large-Scale Applications*
}

\author{
Steven J. Benson ${ }^{\dagger} \quad$ Todd S. Munson ${ }^{\ddagger}$
}

Submitted: October 29, 2018

\begin{abstract}
Discretizations of infinite-dimensional variational inequalities lead to linear and nonlinear complementarity problems with many degrees of freedom. To solve these problems in a parallel computing environment, we propose two active-set methods that solve only one linear system of equations per iteration. The linear solver, preconditioner, and matrix structures can be chosen by the user for a particular application to achieve high parallel performance. The parallel scalability of these methods is demonstrated for some discretizations of infinite-dimensional variational inequalities.
\end{abstract}

\section{Introduction}

Achieving high performance for numerical methods in parallel computing environments demands that the user have the ability to customize algorithms, linear solvers, and data structures for their particular problems. Closed environments or algorithms requiring specific linear algebra constrain the choices available to the user, inevitably leading to inefficiency. This paper concerns a flexible, open environment, the Toolkit for Advanced Optimization, and two algorithms for solving complementarity problems implemented so that the user can exploit problem structure. The benefits of this design are significant reductions in solution time and good parallel scalability on targeted complementarity problems.

Complementarity problems arise in various application areas (see, e.g., 22, [18). In this paper, we are interested particularly in applications arising from infinite-dimensional box-constrained variational inequalities where a discretization of the problem corresponds to a large-scale linear or nonlinear complementarity problem [15, 25]. One example of this type, the journal bearing problem

\footnotetext{
${ }^{*}$ This work was supported by the Mathematical, Information, and Computational Sciences Division subprogram of the Office of Advanced Scientific Computing, Office of Science, U.S. Department of Energy, under Contract W-31-109-Eng-38.

${ }^{\dagger}$ Mathematics and Computer Science Division, Argonne National Laboratory, Argonne, Illinois 60439, benson@mcs.anl.gov

${ }^{\ddagger}$ Mathematics and Computer Science Division, Argonne National Laboratory, Argonne, Illinois 60439, tmunson@mcs.anl.gov
} 
[3] involves determining the pressure distribution of a thin film of lubricant between two circular cylinders. This problem is posed as an elliptic partial differential equation with a free boundary. A finite-difference scheme for solving instances of this problem has $n_{x} n_{y}$ degrees of freedom, where $n_{x}$ and $n_{y}$ are the number of points in the spatial discretizations. The large number of degrees of freedom and structure imposed by the finite-difference scheme implies that high performance can be achieved when solving this problem in a parallel environment. Other applications that can be posed as infinite-dimensional variational inequalities include pricing American options [23, 34, nonlinear obstacle problems [30, and optimal control problems 33].

The Toolkit for Advanced Optimization (TAO) provides a flexible environment for solving optimization and complementarity problems in parallel [8]. Several numerical linear algebra packages have been incorporated into TAO 44, 24, 16. These packages offer many algorithmic choices so that a user can select the most appropriate method for a particular application. Novice users can rely on the provided defaults, while more advanced users have the freedom to select their problem representation and linear algebra to improve parallel performance. All of the source code for TAO can be downloaded from [7, which includes our implementations of several algorithms for solving complementarity problems.

Section 2 discusses the design philosophy of and facilities provided by the Toolkit for Advanced Optimization. The complementarity algorithms used to solve the discretized nonlinear complementarity problems in a parallel computing environment are discussed in Section 3. Two methods were implemented and tested: a semismooth method [11, 27] and a reduced space method similar to those presented in 14, 17. These algorithms are attractive because they

solve only a single system of linear equations per iteration. These solves can be performed by using preconditioned iterative methods [31, taking advantage of the research lavished on numerical linear algebra for solving partial differential equations. Numerical results on the MCPLIB collection [12] of complementarity problems are presented in Section 4 to demonstrate that the methods are reasonably robust on general problems. The parallel performance achieved on several discretizations of infinite-dimensional variational inequalities shows the benefits of customizing the linear algebra: both good parallel performance and scalability are achieved.

\section{Design Philosophy}

Traditionally, the numerical methods used in an algorithm implementation to solve linear systems of equations are chosen by the developer. Since most floating point operations are typically performed by the linear system solver, considerable effort has been expended to select and implement one that is efficient and robust for a diverse collection of applications. Efficiency for particular problem instances has been sacrificed in exchange for robustness and general applicability. However, linear solvers tailored to a particular application may 
save a significant amount of computation and allow the method to find solutions to larger problems than previously possible. While developers choose a linear solver without any knowledge of the application, many applications have structure that can be exploited. The Toolkit for Advanced Optimization (TAO) [7, 9, 8, was specifically designed to allow the user to customize the linear solver to their application so that they can achieve high performance and parallel scalability.

One linear solver choice is an LU factorization, which can be applied to arbitrary nonsingular systems. Many robust implementations have been developed that utilize sparsity while maintaining numerical stability. The complementarity methods in TAO allow for the use of the LU factorizations from LAPACK 1 for dense systems and LUSOL 28 for sparse systems. Nonetheless, these factorizations do not exploit symmetry, strong monotonicity, or other features of the matrix, such as block structure. Furthermore, direct factorizations can impose excessive memory requirements even when the given matrix is sparse. Access to Cholesky factorizations are provided when a symmetric positive definite linear system is solved. In this case, the number of floating-point operations can be reduced by a factor of two, and further gains can be achieved by stable reorderings of the matrix based solely on the sparsity pattern.

Aside from direct methods, many iterative techniques can be applied to solve linear systems of equations. These techniques typically do not impose the memory requirements of direct methods. Two of the most common techniques are GMRES 32 for general nonsingular matrices and the conjugate gradient method 20. for symmetric positive definite matrices. Convergence of these methods can be significantly improved by using a preconditioner, such as an incomplete factorization, which works well on general problems, or an application-specific preconditioner, such as an overlapping additive Schwartz method [5].

In a parallel environment, the cost of message passing magnifies the importance of an appropriate selection of the linear system solver and preconditioner. Parallel implementations of GMRES, conjugate gradients, and many other iterative methods, along with scalable preconditioners such as incomplete factorizations and additive Schwartz methods, are provided in the PETSc toolkit [5, 6]. These linear system solvers use the Message Passing Interface (MPI) 21 for communication between processors.

Providing the user with sufficient flexibility in the choice of linear solver requires careful selection and implementation of the complementarity algorithms. In particular, the interface to the numerical objects, iterative methods and linear algebra, must be separated from their implementations. Object-oriented techniques permit the use of serial or parallel data structures with minimal changes to the interface and allow new implementations of linear system solvers and associated linear algebra to be incorporated. TAO was designed to enable this flexibility and leverages many of the existing parallel linear algebra tools, such as HYPRE 24, PETSc, and Trilinos 16. The optimization methods in TAO, which include the complementarity methods and solvers to minimize an objective function with bounds on the variables, are written to scale on parallel 
machines and expose many of the algorithmic details, such as the linear solver, to the user. This design philosophy assumes that the user has knowledge of an application that can be used to improve the parallel performance.

\section{Complementarity Algorithms}

The finite-dimensional nonlinear complementarity problem defined by a given function $F: \Re^{n} \rightarrow \Re^{n}$ is to calculate an $x^{*} \in \Re^{n}$ such that $x^{*} \geq 0, F\left(x^{*}\right) \geq 0$, and $\left\langle x^{*}, F\left(x^{*}\right)\right\rangle=0$. The algorithms implemented in this study use the current iterate to define an active set, solve a reduced system to calculate a direction, and then perform a line search to compute a new iterate that sufficiently decreases a merit function.

\subsection{Active-Set Semismooth Method}

The semismooth algorithm reformulates a given complementarity problem as a nonsmooth system of equations satisfying a semismooth property by using an NCP function. The resulting nonsmooth system of equations is solved with Newton's method where an element of the B-subdifferential plays the role of the Jacobian matrix in the direction calculation. The active-set semismooth method implemented in TAO is based on this idea.

Mathematically, a function $\phi: \Re^{2} \rightarrow \Re$ is said to be an NCP function if $\phi(a, b)=0$ if and only if $a \geq 0, b \geq 0$, and $a b=0$. By defining

$$
\Phi(x):=\left[\begin{array}{c}
\phi\left(x_{1}, F_{1}(x)\right) \\
\phi\left(x_{2}, F_{2}(x)\right) \\
\vdots \\
\phi\left(x_{n}, F_{n}(x)\right)
\end{array}\right]
$$

for any NCP function $\phi$, the nonlinear complementarity problem can be reformulated as finding an $x^{*} \in \Re^{n}$ such that $\Phi\left(x^{*}\right)=0$. In order to globalize a Newton method for solving this system of equations, the merit function

$$
\Psi(x):=\frac{1}{2}\|\Phi(x)\|_{2}^{2}
$$

is typically used in a line search.

The Fischer-Burmeister function 19,

$$
\phi_{F B}(a, b):=a+b-\sqrt{a^{2}+b^{2}}
$$

is one NCP function, where $\Phi_{F B}$ denotes the reformulation of the nonlinear complementarity problem implied by using $\phi_{F B}$. Even though $\Phi_{F B}$ is not continuously differentiable, a Newton method can still be constructed for finding $\Phi_{F B}(x)=0$. 
The function $\Phi_{F B}$ is differentiable almost everywhere and therefore admits a B-subdifferential [29]

$$
\partial_{B} \Phi_{F B}(x):=\left\{H \in \Re^{n \times n} \mid \exists\left\{x^{k}\right\} \subseteq D_{\Phi_{F B}} \text { with } \lim _{x^{k} \rightarrow x} \nabla \Phi_{F B}\left(x^{k}\right)=H\right\},
$$

where $D_{\Phi_{F B}}$ denotes the set of points where $\Phi_{F B}$ is differentiable. In particular,

$$
\partial_{B} \Phi_{F B}(x) \subseteq\left\{D_{a}(x)+D_{b}(x) \nabla F(x)\right\}
$$

for nonnegative diagonal matrices $D_{a}(x)$ and $D_{b}(x)$ defined componentwise as follows [17:

(a) If $\left\|\left(x_{i}, F_{i}(x)\right)\right\|_{2}>0$, then

$$
\begin{aligned}
{\left[D_{a}(x)\right]_{i, i} } & :=1-\frac{x_{i}}{\left\|\left(x_{i}, F_{i}(x)\right)\right\|_{2}} \\
{\left[D_{b}(x)\right]_{i, i} } & :=1-\frac{F_{i}(x)}{\left\|\left(x_{i}, F_{i}(x)\right)\right\|_{2}} .
\end{aligned}
$$

(b) Otherwise

$$
\left(\left[D_{a}(x)\right]_{i, i},\left[D_{b}(x)\right]_{i, i}\right) \in\left\{(1-\alpha, 1-\beta) \mid\|(\alpha, \beta)\|_{2} \leq 1\right\} .
$$

Furthermore, the merit function, $\Psi_{F B}(x):=\frac{1}{2}\left\|\Phi_{F B}(x)\right\|_{2}^{2}$ is continuously differentiable with $\nabla \Psi_{F B}(x)=H^{T} \Phi_{F B}(x)$ for any $H \in \partial_{B} \Phi_{F B}(x)$.

The main computational task in a semismooth Newton method [1] for solving the nonsmooth system of equations $\Phi_{F B}(x)=0$ is to calculate a direction by solving the linear system of equations

$$
H^{k} d^{k}=-\Phi_{F B}\left(x^{k}\right)
$$

where $H^{k}$ is any element of $\partial_{B} \Phi_{F B}\left(x^{k}\right)$. An Armijo line search [2] along this direction is then applied to obtain a new iterate that sufficiently decreases the merit function. When the full system is solved to find the Newton direction, one must use a method suitable for nonsymmetric matrices because of the row scaling implied by the characterization of the B-subdifferential. Alternatively, one can solve a reduced system, where only a nonnegative diagonal perturbation is made to $\nabla F(x)$. This reduced system will be symmetric whenever $\nabla F(x)$ is symmetric, removing the restrictions placed on the linear solver for the full-space system.

The reduced system is obtained by selecting active and inactive sets of constraints. For a fixed $0 \leq \epsilon<1$, define

$$
\begin{aligned}
& \mathcal{A}(x):=\left\{i \in\{1, \ldots, n\} \mid\left[D_{b}(x)\right]_{i, i} \leq \epsilon\right\} \\
& \mathcal{I}(x):=\left\{i \in\{1, \ldots, n\} \mid\left[D_{b}(x)\right]_{i, i}>\epsilon\right\},
\end{aligned}
$$


where $\mathcal{A}(x)$ denotes the active constraints at $x$ and $\mathcal{I}(x)$ the inactive constraints. The characterization of the B-subdifferential can be used to show that

$$
i \in \mathcal{A}(x) \Rightarrow x_{i} \leq \kappa F_{i}(x)
$$

for some $\kappa>0$. The latter characterization is used by the active-set method in in [17, where they show that for all $x$ in a sufficiently small neighborhood of $x^{*}$, the strongly active components are correctly identified.

We are now ready to state our active-set semismooth algorithm.

Algorithm 3.1 Active-Set Semismooth Method

1. Let $F: \Re^{n} \rightarrow \Re^{n}, x^{0} \in \Re^{n}, \epsilon \in[0,1), \rho>0, p>2, \beta<1$, and $\sigma \in\left(0, \frac{1}{2}\right)$ be given. Set $k=0$.

2. If $\left\|\Phi\left(x^{k}\right)\right\|_{2} \leq$ tol, then stop.

3. Otherwise, choose $D_{a}\left(x^{k}\right)$ and $D_{b}\left(x^{k}\right)$ so that

$$
D_{a}\left(x^{k}\right)+D_{b}\left(x^{k}\right) \nabla F\left(x^{k}\right) \in \partial_{B} \Phi_{F B}\left(x^{k}\right),
$$

and determine $\mathcal{A}^{k}:=\mathcal{A}\left(x^{k}\right)$ and $\mathcal{I}^{k}:=\mathcal{I}\left(x^{k}\right)$.

4. Let

$$
d_{\mathcal{A}^{k}}^{k}=-\left[D_{a}\left(x^{k}\right)\right]_{\mathcal{A}^{k}, \mathcal{A}^{k}}^{-1} \Phi_{F B}\left(x^{k}\right)_{\mathcal{A}^{k}},
$$

and approximately solve the reduced system

$$
\begin{array}{r}
\left(\left[D_{b}\left(x^{k}\right)\right]_{\mathcal{I}^{k}, \mathcal{I}^{k}}^{-1}\left[D_{a}\left(x^{k}\right)\right]_{\mathcal{I}^{k}, \mathcal{I}^{k}}+\left[\nabla F\left(x^{k}\right)\right]_{\mathcal{I}^{k}, \mathcal{I}^{k}}\right) d_{\mathcal{I}^{k}}^{k}= \\
-\left[D_{b}\left(x^{k}\right)\right]_{\mathcal{I}^{k}, \mathcal{I}^{k}}^{-1} \Phi_{F B}\left(x^{k}\right)_{\mathcal{I}^{k}}-\left[\nabla F\left(x^{k}\right)\right]_{\mathcal{I}^{k}, \mathcal{A}^{k}} d_{\mathcal{A}^{k}}^{k}
\end{array}
$$

to find $d_{\mathcal{I}^{k}}^{k}$.

5. If the descent test

$$
\nabla \Psi_{F B}\left(x^{k}\right)^{T} d^{k} \leq-\rho\left\|d^{k}\right\|_{2}^{p}
$$

is not satisfied, set $d^{k}=-\nabla \Psi_{F B}\left(x^{k}\right)$.

6. Calculate the smallest $i \in\{0,1, \ldots\}$ such that

$$
\Psi_{F B}\left(x^{k}+\beta^{i} d^{k}\right) \leq \Psi_{F B}\left(x^{k}\right)+\sigma \beta^{k} \nabla \Psi_{F B}\left(x^{k}\right)^{T} d^{k},
$$

and set $x^{k+1}=x^{k}+\beta^{i} d^{k}$.

7. Set $k=k+1$, and go to Step 2.

The advantages of this active-set method are that the linear systems of equations solved to calculate the Newton directions are of a reduced size and they remain symmetric, positive definite if $\nabla F(x)$ is symmetric, positive definite. Therefore, the choice of preconditioner and iterative method is not restricted to nonsymmetric methods. 
The implementation of the active-set method in TAO uses $\rho=10^{-10}, p=$ 2.1, $\beta=\frac{1}{2}$, and $\sigma=10^{-4}$. The value of $\epsilon$ is dynamically chosen as

$$
\epsilon\left(x^{k}\right)=\frac{\min \left\{\frac{1}{2}\left\|\Phi\left(x^{k}\right)\right\|_{2}^{2}, 10^{-2}\right\}}{1+\left\|\nabla F\left(x^{k}\right)\right\|_{1}} .
$$

This choice forces the active-set identification to go to zero as we approach a solution to the complementarity problem and deals with possible scaling problems with the Jacobian of $F$, since small values for $\left[D_{b}\right]_{i, i}$ can still have an effect on the direction calculation when the Jacobian is poorly scaled. For mixed complementarity problems where we have both finite lower and upper bounds, the Billups formulation is used [10]. The same active-set identification technique is used with this formulation.

\subsection{Reduced-Space Method}

The reduced-space method implemented in TAO also selects an active set and solves a reduced linear system of equations to calculate a direction. The iterates in this method remain within the variable bounds, while no such guarantee is made with the active-set semismooth method. This method is motivated by the simplicity of implementation in a distributed memory computing environment and the computational efficiency of similar methods observed on several classes of problems [14].

The active and inactive sets used within the reduced-space method are defined as

$$
\begin{gathered}
\mathcal{A}(x):=\left\{i \in\{1, \ldots, n\} \mid x_{i}=0 \text { and } F_{i}(x)>0\right\} \\
\mathcal{I}(x):=\left\{i \in\{1, \ldots, n\} \mid x_{i}>0 \text { or } F_{i}(x) \leq 0\right\} .
\end{gathered}
$$

The active set denotes the variables where the lower bound is active and the function value can be ignored. The inactive set contains the remainder of the variables. At every iteration of the reduced-space method a direction is calculated by approximately solving the linear system equation

$$
\left[\nabla F\left(x^{k}\right)\right]_{\mathcal{I}^{k}, \mathcal{I}^{k}} d_{\mathcal{I}^{k}}=-F_{\mathcal{I}^{k}}\left(x^{k}\right)
$$

and setting $d_{\mathcal{A}^{k}}$ to zero.

A projected line search is then applied to generate the next iterate $x^{k+1}$ such that

$$
x^{k+1}=\pi\left[x^{k}+\alpha d^{k}\right],
$$

where $\pi$ is the projection onto the variable bounds. The step size $\alpha$ is chosen such that $\left\|F_{\Omega}\left(\pi\left[x^{k}+\alpha d^{k}\right]\right)\right\|_{2} \leq(1-\sigma \alpha)\left\|F_{\Omega}(x)\right\|_{2}$, where $F_{\Omega}(x)$ is defined component-wise by

$$
\left[F_{\Omega}(x)\right]_{i}=\left\{\begin{array}{lll}
F_{i}(x) & \text { if } & x_{i}>0 \\
\min \left\{F_{i}(x), 0\right\} & \text { if } & x_{i}=0 .
\end{array}\right.
$$


The line search tries step lengths $\alpha=\beta^{j}$ for $\beta \in(0,1)$ and positive integers $j$ such that $\beta^{j}>\gamma$. There are no guarantees the direction calculated is a descent direction, so the line search can terminate with either a new point of sufficient improvement or a minimum step length. The implementation uses $\sigma=10^{-4}$, $\beta=0.5$, and a minimum stepsize of $\gamma=10^{-12}$ for the parameter choices.

If the line search fails to identify such a point, this method performs the same line search in the direction $d^{k}=-F\left(x^{k}\right)$. The algorithm continues until there is a second failure in the line search or a stationary point has been found.

The complete reduced-space algorithm follows.

\section{Algorithm 3.2 Active-Set Reduced Space Method}

1. Let $F: \Re^{n} \rightarrow \Re^{n}$ and $x^{0} \in \Re_{+}^{n}$ be given, and set $k=0$.

2. If $\left\|F_{\Omega}\left(x^{k}\right)\right\|_{2} \leq$ tol, then stop.

3. Let

$$
\begin{gathered}
\mathcal{A}(x):=\left\{i \in\{1, \ldots, n\} \mid x_{i}=0 \text { and } F_{i}(x)>0\right\} \\
\mathcal{I}(x):=\left\{i \in\{1, \ldots, n\} \mid x_{i}>0 \text { or } F_{i}(x) \leq 0\right\},
\end{gathered}
$$

set $d_{\mathcal{A}^{k}}=0$, and approximately solve the linear system

$$
\left[\nabla F\left(x^{k}\right)\right]_{\mathcal{I}^{k}, \mathcal{I}^{k}} d_{\mathcal{I}^{k}}=-F_{\mathcal{I}^{k}}\left(x^{k}\right)
$$

to calculate a direction.

4. If possible, calculate the smallest $i \in\{0,1, \ldots, \bar{i}\}$ such that

$$
\left\|F_{\Omega}\left(\pi\left[x^{k}+\beta^{i} d^{k}\right]\right)\right\|_{2} \leq\left(1-\sigma \beta^{i}\right)\left\|F_{\Omega}\left(x^{k}\right)\right\|_{2} .
$$

5. Otherwise, set $d^{k}=-F\left(x^{k}\right)$, and apply the line search. If no such $i$ is found providing sufficient decrease, then stop.

6. Set $x^{k+1}=\pi\left[x^{k}+\beta^{i} d^{k}\right]$ and $k=k+1$, and go to step 2.

Although no convergence results have been proven for the reduced-space method, it has been demonstrated to be effective, especially on monotone applications. The reduced matrix retains symmetry and positive definiteness when they exist in the Jacobian, permitting the use of a symmetric linear solver. Furthermore, this method lends itself to a parallel implementation because it requires only a few numerical operations other than a linear solver.

\section{Computational Results}

The complementarity methods were implemented in and are distributed with the Toolkit for Advanced Optimization. Two series of tests were performed on the implementations: one to check the robustness to verify that the methods work 
on a significant number of general problems, and the other to test the parallel performance and scalability of the methods on some trial infinite-dimensional variational inequalities. The latter tests also validate the merits of our design philosophy by showing that the customization of the linear system solver does have a significant effect on the overall solution time. For all of these tests, an upper limit of 100 linear system solves was placed on the methods.

\subsection{Robustness}

We ran the implemented methods on the complementarity problems contained in the MCPLIB collection [12 to demonstrate robustness on a diverse set of problems, including many small complementarity problems and some nonlinear obstacle problems and optimal control problems. These computational tests were performed on Linux workstation containing a Pentium 4 processor with a clock speed of $1.8 \mathrm{GHz}$ and $512 \mathrm{MB}$ of RAM.

In order to test the robustness, we ran the methods with LUSOL as the selected linear system solver. This LU factorization routine is used by several optimization and complementarity packages, including MINOS 28] and PATH [13], and is known to be robust and efficient in a serial environment. We chose to use a direct factorization for this test to eliminate possible problems with the choice of preconditioner, iterative method, and termination tolerances for the inexact linear system solves.

Tables 1 and 2 report the number of successes and failures for each method and model in the test set when using LUSOL. These results indicate that the active-set semismooth method, which solves $73.7 \%$ of the problems, is more robust than the reduced-space method, which solves $65.5 \%$ of the problems. The overall conclusion to be drawn is that both methods solve a significant fraction of these test problems. While these robustness results can be improved by introducing heuristics into the implementations, we prefer to use the standard methods because they are less complicated and work well on the targeted infinite-dimensional variational inequalities.

\subsection{Scalability}

To demonstrate the scalability and performance gains that can be made by selecting appropriate linear system solvers, we tested the complementarity algorithms implemented on some discretizations of infinite-dimensional variational inequalities. All of the computational tests in this subsection were performed on a Linux cluster composed of 350 Pentium Xeon processors with a clock speed of $2.4 \mathrm{GHz}$. Each node has a minimum of $1 \mathrm{~GB}$ of RAM is connected by a Myrinet 2000 network.

One benchmark application is the journal bearing model, a variational problem over a two-dimensional region. This problem arises in the determination of the pressure distribution in a thin film of lubricant between two circular cylinders. The infinite-dimensional version of this problem is to find a piecewise 
Table 1: Performance of methods on MCPLIB.

\begin{tabular}{|c|c|c|c|c|}
\hline \multirow[b]{2}{*}{ Problem } & \multicolumn{2}{|c|}{ Active-Set Method } & \multicolumn{2}{|c|}{ Reduced-Space Method } \\
\hline & Successes & Failures & Successes & Failures \\
\hline ahn & 1 & 0 & 1 & 0 \\
\hline badfree & 1 & 0 & 1 & 0 \\
\hline baihaung & 1 & 0 & 1 & 0 \\
\hline bert_oc & 4 & 0 & 4 & 0 \\
\hline bertsekas & 6 & 0 & 3 & 3 \\
\hline billups & 0 & 3 & 0 & 3 \\
\hline bishop & 0 & 1 & 0 & 1 \\
\hline bratu & 0 & 1 & 1 & 0 \\
\hline cammcf & 0 & 1 & 0 & 1 \\
\hline cgereg & 2 & 20 & 14 & 8 \\
\hline choi & 1 & 0 & 1 & 0 \\
\hline colvdual & 2 & 2 & 2 & 2 \\
\hline colvnlp & 6 & 0 & 6 & 0 \\
\hline cycle & 1 & 0 & 1 & 0 \\
\hline danny & 6 & 2 & 6 & 2 \\
\hline degen & 1 & 0 & 1 & 0 \\
\hline dirkse & 1 & 1 & 1 & 1 \\
\hline duopoly & 0 & 1 & 0 & 1 \\
\hline eckstein & 1 & 0 & 0 & 1 \\
\hline ehl & 2 & 10 & 6 & 6 \\
\hline electric & 0 & 1 & 0 & 1 \\
\hline explcp & 1 & 0 & 0 & 1 \\
\hline exros & 1 & 4 & 1 & 4 \\
\hline ferrralph & 2 & 0 & 2 & 0 \\
\hline finance & 60 & 0 & 59 & 1 \\
\hline fixedpt & 0 & 2 & 0 & 2 \\
\hline force & 0 & 2 & 0 & 2 \\
\hline freebert & 3 & 4 & 5 & 2 \\
\hline fried & 5 & 5 & 6 & 4 \\
\hline friedms & 1 & 0 & 1 & 0 \\
\hline gafni & 3 & 0 & 3 & 0 \\
\hline games & 18 & 7 & 0 & 25 \\
\hline golanmcp & 0 & 1 & 1 & 0 \\
\hline hanskoop & 7 & 3 & 5 & 5 \\
\hline hydroc & 2 & 0 & 2 & 0 \\
\hline jel & 2 & 0 & 2 & 0 \\
\hline jiangqi & 3 & 0 & 3 & 0 \\
\hline josephy & 8 & 0 & 5 & 3 \\
\hline kanzow & 7 & 0 & 7 & 0 \\
\hline kojshin & 8 & 0 & 5 & 3 \\
\hline kyh & 0 & 4 & 0 & 4 \\
\hline lincont & 0 & 1 & 0 & 1 \\
\hline lstest & 0 & 1 & 0 & 1 \\
\hline leyffer & 1 & 0 & 1 & 0 \\
\hline
\end{tabular}


Table 2: Performance of methods on MCPLIB.

\begin{tabular}{|c|c|c|c|c|}
\hline \multirow[b]{2}{*}{ Problem } & \multicolumn{2}{|c|}{ Active-Set Method } & \multicolumn{2}{|c|}{ Reduced-Space Method } \\
\hline & Successes & Failures & Successes & Failures \\
\hline mathi & 13 & 0 & 13 & 0 \\
\hline methan & 1 & 0 & 1 & 0 \\
\hline mr5mcf & 0 & 1 & 0 & 1 \\
\hline munson & 3 & 1 & 3 & 1 \\
\hline nash & 4 & 0 & 4 & 0 \\
\hline ne-hard & 0 & 1 & 1 & 0 \\
\hline obstacle & 8 & 0 & 8 & 0 \\
\hline optcont & 5 & 0 & 5 & 0 \\
\hline pgvon & 0 & 12 & 2 & 10 \\
\hline pies & 1 & 0 & 0 & 1 \\
\hline pizer & 1 & 3 & 3 & 1 \\
\hline powell & 5 & 1 & 3 & 3 \\
\hline powellmcp & 6 & 0 & 6 & 0 \\
\hline poz & 6 & 0 & 6 & 0 \\
\hline qp & 1 & 0 & 1 & 0 \\
\hline runge & 3 & 4 & 0 & 7 \\
\hline scarf & 9 & 3 & 6 & 6 \\
\hline shansim & 1 & 0 & 1 & 0 \\
\hline shubik & 11 & 37 & 10 & 38 \\
\hline simple-ex & 0 & 1 & 0 & 1 \\
\hline simple-red & 1 & 0 & 1 & 0 \\
\hline spillmcp & 0 & 1 & 0 & 1 \\
\hline sppe & 2 & 1 & 3 & 0 \\
\hline sun & 1 & 0 & 1 & 0 \\
\hline taji & 12 & 0 & 12 & 0 \\
\hline tiebout & 0 & 6 & 0 & 6 \\
\hline tinloi & 64 & 0 & 63 & 1 \\
\hline tinsmall & 63 & 1 & 63 & 1 \\
\hline titan & 1 & 1 & 1 & 1 \\
\hline tobin & 4 & 0 & 4 & 0 \\
\hline tqbilat & 1 & 1 & 2 & 0 \\
\hline trafelas & 0 & 2 & 0 & 2 \\
\hline trig & 2 & 1 & 0 & 3 \\
\hline vonthmcf & 0 & 1 & 0 & 1 \\
\hline xiaohar & 4 & 0 & 3 & 1 \\
\hline $\mathrm{xu}$ & 35 & 0 & 5 & 30 \\
\hline Total & 437 & 156 & 391 & 202 \\
\hline
\end{tabular}




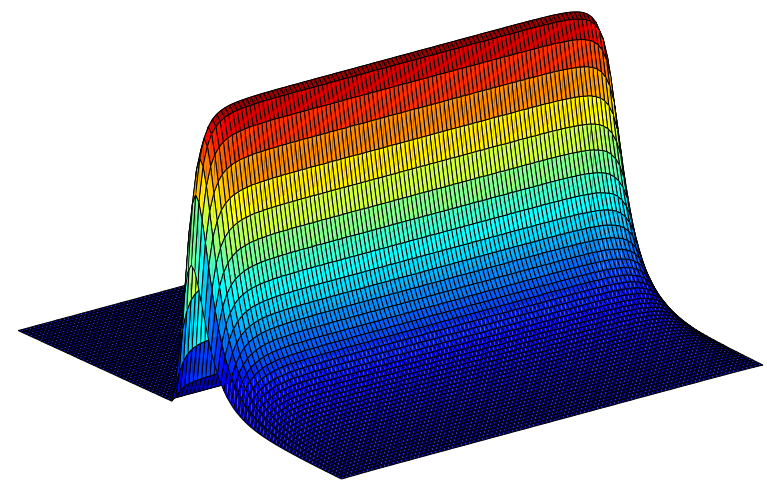

Figure 1: The journal bearing problem with $\varepsilon=0.9$.

continuously differentiable function, $v: \mathcal{D} \mapsto \mathbb{R}$, such that

$$
0 \leq v, w_{q} \Delta v \geq w_{l} \text {, and } v\left[w_{q} \Delta v-w_{l}\right]=0
$$

almost everywhere on $\mathcal{D}$ with $v=0$ on $\partial \mathcal{D}$, where $\mathcal{D}=(0,2 \pi) \times(0,2 b)$ for some constant $b>0$ and

$$
w_{q}\left(\xi_{1}, \xi_{2}\right)=\left(1+\varepsilon \cos \xi_{1}\right)^{3}, \quad w_{l}\left(\xi_{1}, \xi_{2}\right)=\varepsilon \sin \xi_{1}
$$

with $\varepsilon$ in $(0,1)$. The eccentricity parameter, $\varepsilon$, influences, in particular, the difficulty of the problem. Figure 1 shows the solution of the journal bearing problem for $\varepsilon=0.9$. The steep gradient in the solution makes this problem a difficult benchmark. Other elliptic problems tested include the obstacle, elasticplastic torsion, and combustion models from MINPACK-2; see [3] for a complete description of these models.

Discretization of the journal bearing problem with either finite differences or finite elements leads to a standard complementarity problem. The number of variables is $n=n_{x} n_{y}$, where $n_{x}$ and $n_{y}$ are, respectively, the number of grid points in each coordinate direction of the domain $\mathcal{D}$. See for [26] a description of the finite-element discretization.

The number of grid points can be very large. In fact, these problems become so large that the Jacobian matrix cannot even be stored on one machine. Direct factorizations exacerbate the computer memory issues because the fill-in associated with a direct factorization is significant. For this reason, iterative linear solvers are necessary.

The Jacobian of this problem has a sparse, symmetric, and positive definite structure. Considerable improvement can be made by using the conjugate gradient method to solve the linear systems with an incomplete factorization used as a 
preconditioner. Many incomplete factorization preconditioners require nonzeros along the diagonal, and the journal bearing problem satisfies this requirement.

The problem was discretized into 40,000 variables and then solved with three different solvers: LUSOL, a conjugate gradient method with an incomplete LU preconditioner, and a conjugate gradient method with a block Jacobi preconditioner such that each block contains an incomplete LU factorization. As Table 3 shows, use of the conjugate gradient methods with with an appropriate preconditioner saves a significant amount of time. With the reduced-space method, the savings range from $30 \%$ on the combustion problem to over $70 \%$ on the elastic-plastic torsion problem.

Table 3: Solution times for three linear solvers (sec).

\begin{tabular}{|l|ccc|}
\multicolumn{1}{l|}{ Problem } & LUSOL & CG - 1 P & CG - 2 P \\
\hline J Bearing & 278 & 136 & 99 \\
EP Torsion & 172 & 50 & 34.6 \\
Obstacle & 49 & 18.4 & 14.3 \\
Combustion & 29.8 & 20.2 & 14.1 \\
\hline
\end{tabular}

To demonstrate the parallel efficiency of the complementarity methods, we wrote parallel implementations of the elastic-plastic torsion and obstacle problems using the grid management facilities of PETSc, which relies on MPI [21] for communications between processors. PETSc provides support for discretizing the rectangular region $\mathcal{D}$, partitioning the surface into multiple regions, and assigning each processor to one of these regions. Each processor computes the function on its subdomain with respect to the variables in its region. A preconditioned conjugate gradient method was used to solve the linear systems generated by the complementarity algorithms. The preconditioner was a block Jacobi preconditioner with incomplete LU factorization. In the reduced-space method, the relative tolerance used during the linear solve was 0.01 . The results are summarized in Table 4 when using 1-64 processors.

The overall efficiency of our implementation is shown in Figure 2(a) Each bar indicates the performance of the active-set semismooth method relative to its performance on two processors. This number is the ratio of two times the execution time using two processors and the number of processors multiplied by the time needed to solve the problem using one of those processors. With this metric, the overall parallel efficiency active-set semismooth method on the obstacle problems using 16 processors was over $83 \%$, and the overall parallel efficiency using 64 processors was over $60 \%$. The overall efficiency of the reduced-space method was similar.

A second set of tests was performed with the mesh refined according the number of processors used to solve the problem. In these tests each processor owned 10, 000 variables. The mesh was $100 \times 100$ when one processor was used and $800 \times 800$ when 64 processors were used. Since the methods require more iterations to solve problems with finer meshes, a comparison of running times 
Table 4: Scalability.

\begin{tabular}{|llll|ccccccc|}
\hline Problem & Solver & $\mathrm{mx}$ & \multicolumn{1}{c|}{$\mathrm{my}$} \\
\hline & & & & 1 & 2 & 4 & 8 & 16 & 32 & 64 \\
\hline Obstacle & R-S & 800 & 800 & 2848 & 1722 & 887 & 484 & 252 & 129 & 71 \\
Obstacle & A-S & 800 & 800 & 975 & 582 & 300 & 168 & 87 & 51 & 30 \\
JBearing & R-S & 800 & 800 & 13597 & 7786 & 7021 & 3555 & 1861 & 930 & 544 \\
JBearing & A-S & 800 & 800 & 14025 & 7770 & 7019 & 3540 & 1973 & 1082 & 648 \\
Eptorsion & R-S & 800 & 800 & 835 & 548 & 287 & 152 & 112 & 58 & 41 \\
Eptorsion & A-S & 800 & 800 & 4744 & 3118 & 1644 & 877 & 463 & 261 & 165 \\
Combustion & R-S & 800 & 800 & 332 & 228 & 122 & 63 & 31 & 15 & 8.6 \\
Combustion & A-S & 800 & 800 & 339 & 233 & 124 & 67 & 32 & 17 & 10.2 \\
\hline
\end{tabular}

would not demonstrate the scalability of the methods. Instead, we used the rate of floating-point operations as the measure of efficiency. Figure 2(b) shows the average number of floating-point operations performed per second on each processor when the reduced space method was used to solve the torsion problem. As the overhead of message passing increases, the rate of computation on each processor decreases. The problem used over 142 MFlops when one processor was used and that rate only decreased to about 124 MFlops when when 64 processors were used. Our parallel efficiency of both methods by this measure often exceeded $80 \%$ on sixty four processors.

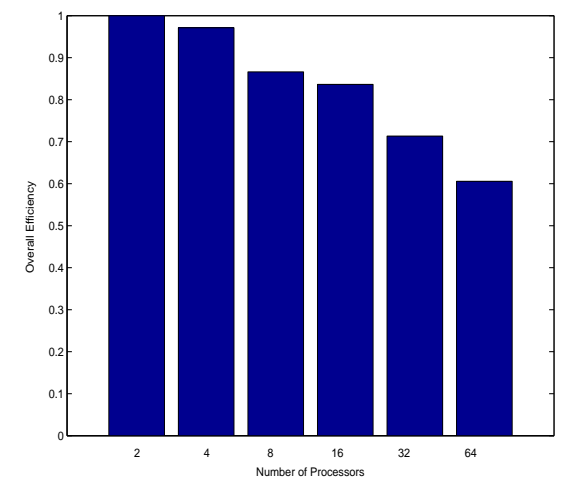

(a) Overall Implementation Efficiency

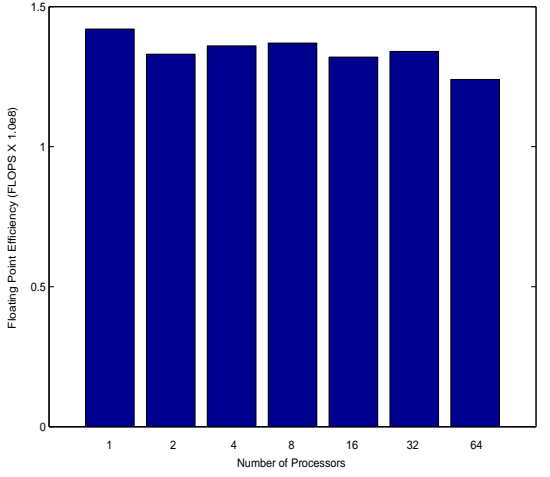

(b) Floating-Point Efficiency

Figure 2: Parallel efficiency on the obstacle problem. 


\section{Conclusion}

The complementarity methods implemented within the Toolkit for Advanced Optimization are reasonably robust and can effectively solve discretized versions of infinite-dimensional variational inequalities. In order to achieve high parallel performance, the methods were implemented so that the user has flexibility in the choice of data structures, linear algebra, and algorithms.

\section{References}

[1] E. Anderson, Z. Bai, C. Bischof, J. Demmel, J. Dongarra, J. Du Cros, A. Greenbaum, S. Hammarling, A. McKenney, S. Ostrouchov, and D. Sorensen. LAPACK User's Guide, second ed. SIAM, Philadelphia, 1995.

[2] L. Armijo. Minimization of functions having Lipschitz-continuous first partial derivatives. Pacific Journal of Mathematics, 16:1-3, 1966.

[3] B. M. Averick, R. G. Carter, J. J. Moré, and G. L. Xue. The MINPACK-2 test problem collection. Technical Report MCS-P153-0692, Argonne National Laboratory, Argonne, Illinois, 1992.

[4] S. Balay, W. Gropp, L. Curfman-McInnes, and B. Smith. PETSc Web page. See http://www.mcs.anl.gov/petsc.

[5] Satish Balay, William D. Gropp, Lois Curfman McInnes, and Barry F. Smith. Efficient management of parallelism in object oriented numerical software libraries. In E. Arge, A. M. Bruaset, and H. P. Langtangen, editors, Modern Software Tools in Scientific Computing, pages 163-202. Birkhauser Press, 1997.

[6] Satish Balay, William D. Gropp, Lois Curfman McInnes, and Barry F. Smith. PETSc 2.0 users manual. Technical Report ANL-95/11 - Revision 2.1.0, Argonne National Laboratory, April 2001.

[7] Steve Benson, Lois Curfman McInnes, and Jorge Moré. Toolkit for Advanced Optimization (TAO) Web page. See http://www.mcs.anl.gov/tao

[8] Steve Benson, Lois Curfman McInnes, and Jorge Moré. TAO users manual. Technical Report ANL/MCS-TM-242, Mathematics and Computer Science Division, Argonne National Laboratory, 2000. See http://www.mcs.anl.gov/tao

[9] Steven J. Benson, Lois Curfman McInnes, and Jorge J. More'. A case study in the performance and scalability of optimization algorithms. ACM Transactions on Mathematical Software, 27(3):361-376, September 2001.

[10] S. C. Billups. Algorithms for Complementarity Problems and Generalized Equations. PhD thesis, University of Wisconsin, Madison, 1995. 
[11] T. De Luca, F. Facchinei, and C. Kanzow. A semismooth equation approach to the solution of nonlinear complementarity problems. Mathematical Programming, 75:407-439, 1996.

[12] S. P. Dirkse and M. C. Ferris. MCPLIB: A collection of nonlinear mixed complementarity problems. Optimization Methods and Software, 5:319-345, 1995.

[13] S. P. Dirkse and M. C. Ferris. The PATH solver: A non-monotone stabilization scheme for mixed complementarity problems. Optimization Methods and Software, 5:123-156, 1995.

[14] S. P. Dirkse and M. C. Ferris. Crash techniques for large-scale complementarity problems. In M. C. Ferris and J. S. Pang, editors, Complementarity and Variational Problems: State of the Art, pages 40-61, Philadelphia, 1997. SIAM.

[15] I. Ekeland and R. Témam. Convex Analysis and Variational Problems. North-Holland, Amsterdam, 1976.

[16] Mike Heroux et al. Trilinos Web page. See http://www.cs.sandia.gov/〜mheroux/Trilinos/doc/TrilinosIntroduction.htm

[17] F. Facchinei and J. Soares. A new merit function for nonlinear complementarity problems and a related algorithm. SIAM Journal on Optimization, $7: 225-247,1997$.

[18] M. C. Ferris and J. S. Pang. Engineering and economic applications of complementarity problems. SIAM Review, 39:669-713, 1997.

[19] A. Fischer. A special Newton-type optimization method. Optimization, 24:269-284, 1992.

[20] G. H. Golub and C. F. Van Loan. Matrix Computations, third ed. The John Hopkins University Press, Baltimore, Maryland, 1996.

[21] William Gropp, Ewing Lusk, and Anthony Skjellum. Using MPI: Portable Parallel Programming with the Message Passing Interface. MIT Press, 1994.

[22] P. T. Harker and J. S. Pang. Finite-dimensional variational inequality and nonlinear complementarity problems: A survey of theory, algorithms and applications. Mathematical Programming, 48:161-220, 1990.

[23] J. Huang and J. S. Pang. Option pricing and linear complementarity. Journal of Computational Finance, 2:31-60, 1998.

[24] hypre: High performance preconditioners. http://www.llnl.gov/CASC/hypre/. 
[25] D. Kinderlehrer and G. Stampacchia. An Introduction to Variational Inequalities and Their Applications. Academic Press, New York, 1980.

[26] J. J. Moré and G. Toraldo. On the solution of large quadratic programming problems with bound constraints. SIAM Journal on Optimization, 1:93$113,1991$.

[27] T. S. Munson, F. Facchinei, M. C. Ferris, A. Fischer, and C. Kanzow. The semismooth algorithm for large scale complementarity problems. INFORMS Journal on Computing, 13:294-311, 2001.

[28] B. A. Murtagh and M. A. Saunders. MINOS 5.0 user's guide. Tech. Rep. SOL 83.20, Stanford University, Stanford, California, 1983.

[29] L. Qi. Convergence analysis of some algorithms for solving nonsmooth equations. Mathematics of Operations Research, 18:227-244, 1993.

[30] J. F. Rodrigues. Obstacle Problems in Mathematical Physics. Elsevier Publishing Company, Amsterdam, 1987.

[31] Y. Saad. Iterative Methods for Sparse Linear Systems. PWS Publishing Company, Boston, 1996.

[32] Y. Saad and M. Schultz. GMRES: A generalized minimal residual algorithm for solving nonsymmetric linear systems. SIAM Journal on Scientific and Statistical Computing, 44:856-869, 1986.

[33] M. Ulbrich. On a nonsmooth Newton method for nonlinear complementarity problems in function space with applications in optimal control. In M. C. Ferris, O.L. Mangasarian, and J. S. Pang, editors, Complementarity: Applications, Algorithms and Extensions, volume 50 of Applied Optimization, pages 341-360, Dordrecht, The Netherlands, 2001. Kluwer Academic Publishers.

[34] P. Wilmott, J. Dewynne, and S. Howison. Option Pricing. Oxford Financial Press, Oxford, England, 1993.

The submitted manuscript has been created by
the University of Chicago as Operator of Argonne
National Laboratory ("Argonne") under Contract
No. W-31-109-ENG-38 with the U.S. Department
of Energy. The U.S. Government retains for it-
self, and others acting on its behalf, a paid-up,
nonexclusive, irrevocable worldwide license in said
article to reproduce, prepare derivative works, dis-
tribute copies to the public, and perform publicly
and display publicly, by or on behalf of the Gov-
ernment.




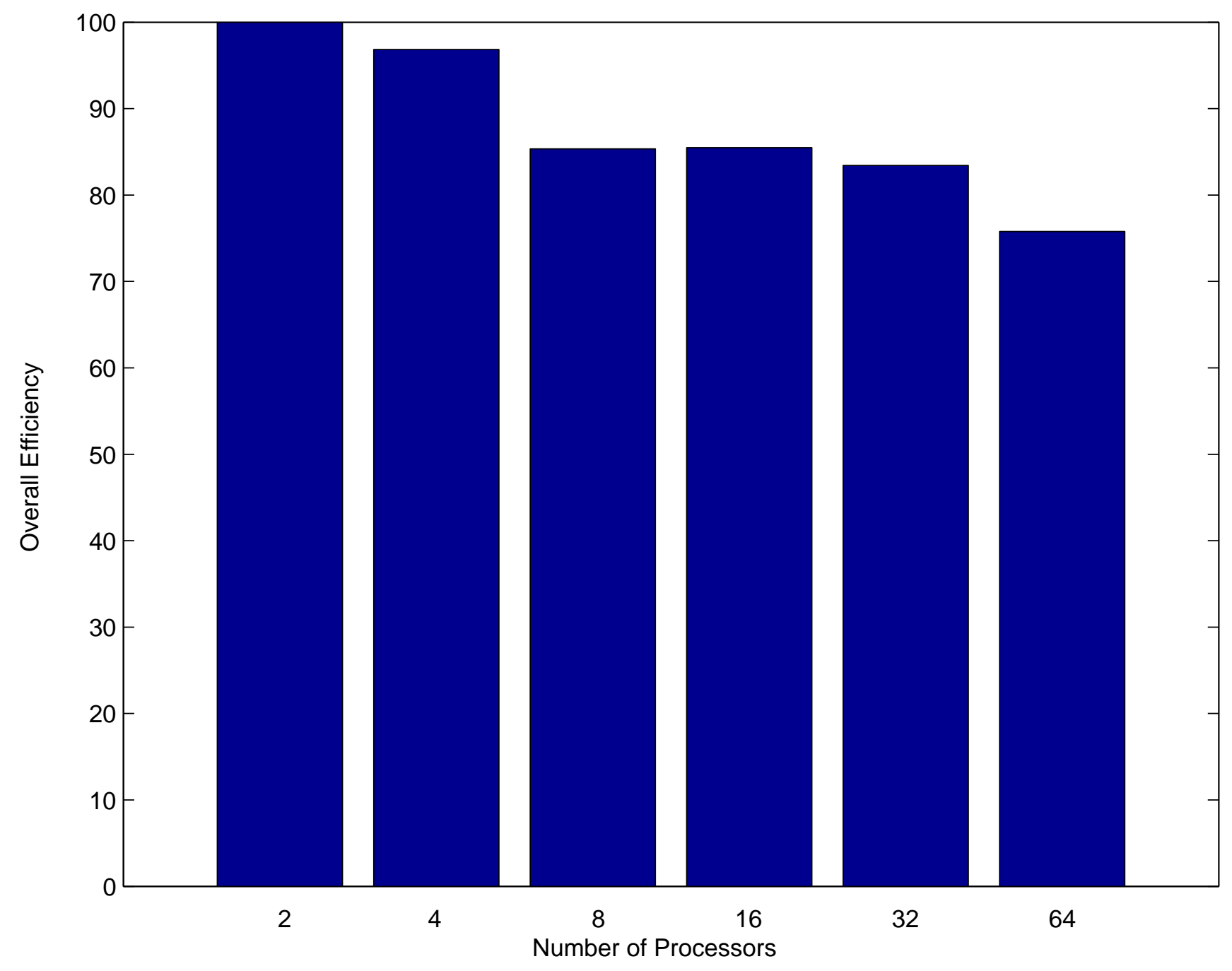




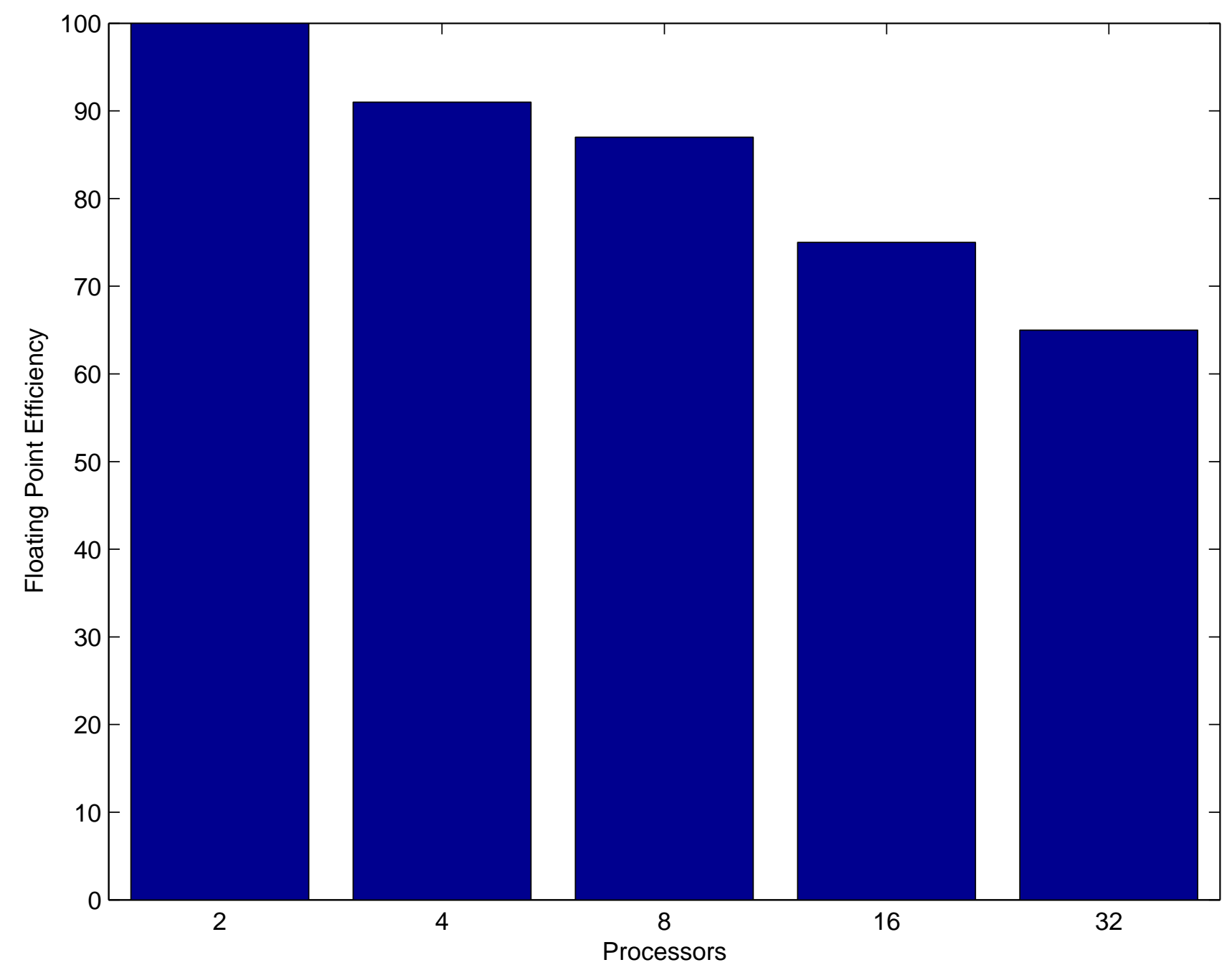




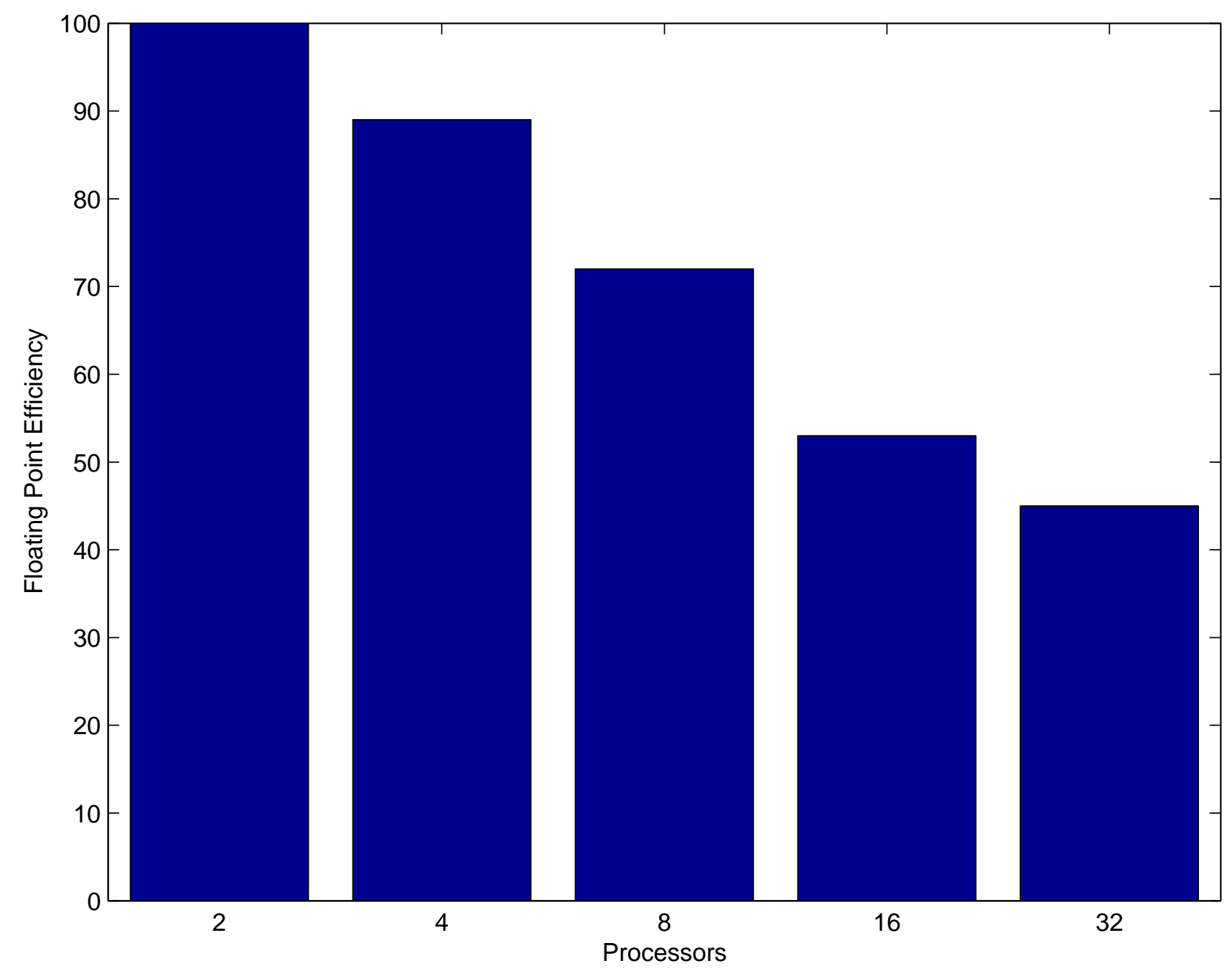

